\title{
Multi-Fingered Haptic Interface Robot and Its Application Systems
}

\author{
Haruhisa Kawasaki \\ Gifu University, 1-1 Yanagido, Gifu 501-1193, Japan \\ h_kawasa@gifu-u.ac.jp
}

Keywords: Haptics, robot, virtual reality, redundant control, FEM, haptic rendering

\begin{abstract}
Haptic interfaces that represent force and tactile feeling have been utilized in the areas of telemanipulation, interaction with microscale and nanoscale phenomena, and medical training and evaluation, to mention only some applications. A multi-fingered haptic interface has greater potential for these kinds of applications than does a singlepoint haptic interface. We developed a five-fingered haptic interface robot named HIRO II, which consists of a hand with 15 dof and an arm with 6 dof. The following research issues are presented: the design method of mechanism, an interface control that takes the redundancy of the mechanism into consideration, physical simulation including frictional force and moment, a haptic rendering with a deformable object, the system architecture and two application systems--a future science encyclopedia and a VR breast palpation system.
\end{abstract}

\section{Introduction}

Haptic interfaces that represent force and tactile feeling have been utilized in the areas of telemanipulation [1]-[2], interaction with microscale and nanoscale phenomena [3], and medical training and evaluation [4]-[5], to mention only some applications. A multi-fingered haptic interface has a greater potential for these kinds of applications than does a singlepoint haptic interface. Several multi-fingered haptic interfaces [6]-[12] have so far been developed. One consisting of an arm and fingers [10]-[12] can be used in a wide space. However, the issue of developing a haptic interface to be used opposite the human hand has so far not been addressed. Such a haptic interface must be safe, function in a wide operation space, and represent the weight of virtual objects as well as force at the contact points. In addition, it should not have an oppressive feeling when it is attached to a person, and it should not represent its own weight.

In response to these issues, we previously developed a three-fingered haptic interface named HIRO [13]. HIRO has demonstrated its suitability as a multi-fingered haptic interface for use opposite a person but is limited by having only three fingers. The next version, HIRO II [14], has five fingers. To develop a virtual reality (VR) simulation system using the multi-fingered haptic interface, the following issues have been studied by our group: the design method of the mechanism including an optimum finger layout, an interface control that takes the redundancy of the mechanism into consideration, physical simulation including frictional force and moment, haptic rendering with a deformable object, the system architecture consisting of a real-time controller and VR simulation, and two application systems--a future science encyclopedia and a VR breast palpation system.

This keynote article presents the design and control of the five-fingered haptic interface to be used opposite a human hand and the above-mentioned technical developments used to integrate a VR simulation system. Moreover, we present the two applications--the future science encyclopedia and the VR breast palpation system--which have been developed to verify our proposal and demonstrate the haptic interface HIRO II.

\section{Design method of the mechanism}

Multi-fingered haptic interfaces joined to the arm can provide a wide operation space. However, most of them are mounted on the back of the human hand like the CyberForce [12]. Fixing the haptic interface to the hand gives the operator an oppressive feeling since the interface binds the 
human hand firmly. In order to reduce the oppressive feeling and increase safety, we developed a new Haptic Interface RObot named HIRO II to represent force feeling to all fingers of the human hand. Fig. 1 shows the developed five-fingered haptic interface connected to the five fingers of an operator's hand. It consists of an interface arm, a haptic hand with five haptic fingers, and a controller. When the operator moves his/her hand, the haptic interface follows the motion of operator's fingers and represents the force feeling. The operator feels just a little oppressive feeling because the part coupled between the human hand and the haptic interface is limited to his/her fingertips.

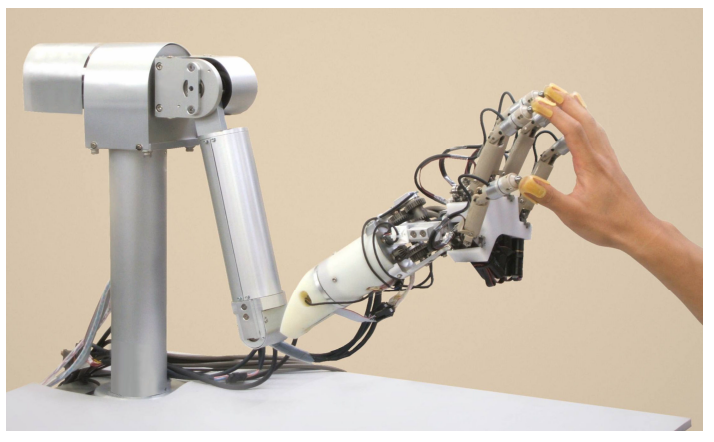

Fig. 1 The Developed five-fingered haptic interface: HIRO II

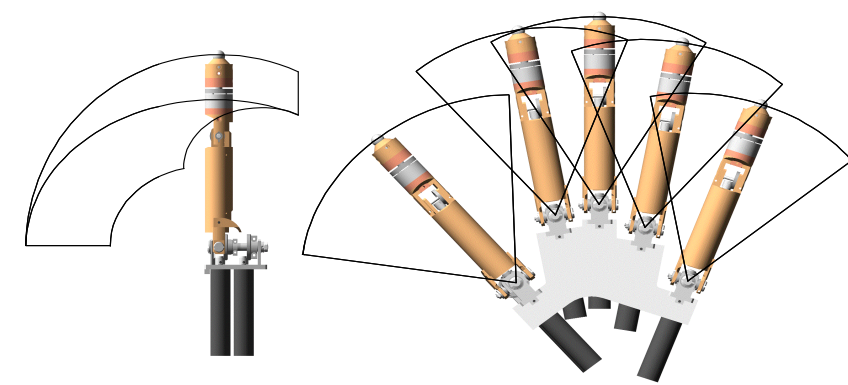

(1) Thumb (2) Hand

Fig. 2 Workspaces of thumb and hand

The developed haptic finger is designed based on an anthropomorphic robot hand named Gifu Hand III [15]. Each finger has 3 joints allowing 3 DOF. The first joint, relative to the hand base, allows abduction/adduction. The second and third joints allow flexion/extension. All joints are driven by DC servomotors with gear transmissions and rotary encoders. Workspaces of the thumb and hand are shown in Fig. 2(1) and (2). The volumes of the workspace of the thumb and fingers are 713 and $535\left[\mathrm{~mm}^{3}\right]$, respectively. The thumb is designed to work in a wide space because the workspace of the human thumb is larger than that of the other fingers. The finger layout of the haptic hand is designed to maximize the volume formed by intersecting workspaces of the haptic and human fingers. For example, this volume for the haptic and human index fingers has its maximum at the posture shown in the Fig. 3. The haptic index finger was designed based on statistical data for Japanese males. The position of fingers in the haptic hand was designed from a consideration of this geometrical relation.

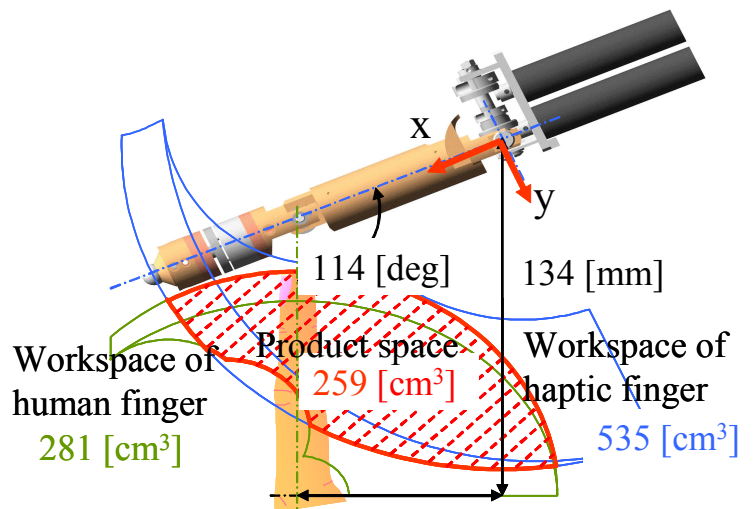

$67[\mathrm{~mm}]$

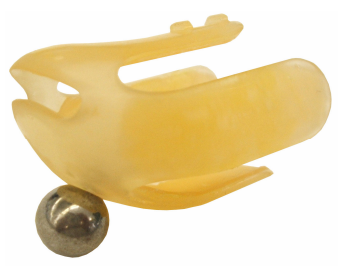

Fig. 4 Finger holder

Fig.3 Optimum pose of the haptic finger for the human finger

In order to read the finger loading, the 6-axes force sensor (NANO sensor by BL AUTOTEC. LTD.) was installed in the second link of each finger. To manipulate the haptic interface, the user has to wear a finger holder on his/her fingertips (see Fig. 4). This finger holder has a sphere which, when attached to the permanent magnet at the force sensor tip, forms a passive spherical joint, which has two roles. One role is adjusting the differences between the orientation of the human and 
haptic fingers. Each human finger has 6 DOF, while each haptic finger has 3 DOF. Hence, an additional passive $3 \mathrm{DOF}$ are needed. The second role of the spherical joint is to ensure that the operator can remove his fingers from the haptic interface when it malfunctions. The suction force by the permanent magnet is $5[\mathrm{~N}]$.

The maximum output forces at a fingertip in the finger coordinate system are 3.4, 3.4, and 6.5 $[\mathrm{N}]$ along the $\mathrm{X}, \mathrm{Y}$ and $\mathrm{Z}$ axes, respectively (see Fig. 3). The maximum fingertip velocities $\mathrm{v}_{\mathrm{x}}, \mathrm{v}_{\mathrm{y}}, \mathrm{v}_{\mathrm{z}}$ are $0.43,0.43$, and $0.23[\mathrm{~m} / \mathrm{s}]$, respectively. The interface arm with 6 DOF is designed to be as close as possible to the human arm in geometry and motion ability. The lengths of the upper arm and the forearm are 0.3 and 0.31 [m], respectively. The arm joints are actuated by AC servomotors equipped with rotary encoders and gear transmissions. As a haptic interface, the combination of the arm and hand makes a wide work space.

\section{Interface control}

The hand posture is nonunique because of the redundancy of the HIRO II. Two approaches that take the redundancy into account have been researched.

Hand manipulability-based control. One control combines finger force control and arm position control [13]. Each finger is controlled independently by a PI control using a force error at the fingertips with a gravitation compensator. In arm position control, a desired hand posture is determined to maximize the hand manipulability measure to respond to an operator's various hand poses. The hand manipulability measure is defined by

$$
C P I=\sum_{i=1}^{5}\left(\alpha_{i} \sqrt{\operatorname{det}\left(\boldsymbol{J}_{F i}^{T}{ }^{T} \boldsymbol{J}_{F i}\right)}+\beta_{i} P_{i}\right)
$$

where $\alpha_{i}$ and $\beta_{i}$ are weighting coefficients, $\boldsymbol{J}_{F i}$ is a kinematic Jacobian of the $i$-th finger and $P_{i}$ is a penalty function of the $i$-th finger to keep the joint angle within the range of movement. This control method may cause a large arm motion even though the operator's finger motions are slight. This excessive motion sometimes confuses the operator because it creates the illusion that the device is malfunctioning. To represent haptic feeling in a wide operation space and reduce the operator's sense of unease, a modified hand manipulability measure has been presented as follows [16]:

$$
C P I=\sum_{i=1}^{5}\left(\alpha_{i} \sqrt{\operatorname{det}\left(\boldsymbol{J}_{F i}{ }^{T} \boldsymbol{J}_{F i}\right)}+\beta_{i} P_{i}\right)-\frac{1}{2}\left(\boldsymbol{q}_{A d}-\boldsymbol{q}_{A}\right)^{T} \Gamma\left(\boldsymbol{q}_{A d}-\boldsymbol{q}_{A}\right)
$$

where $\boldsymbol{q}_{A} \in R^{6}$ is the current arm joint angle vector, $\boldsymbol{q}_{A d} \in R^{6}$ is a desired arm joint angle vector, and $\Gamma>0$ is a weighting matrix. The desired arm joint angle is defined so as to maximize equation (2) under a constraint condition in which the five haptic fingertip positions $\boldsymbol{q}_{F}$ are fixed to the operator's fingertip positions. The optimum arm joint angle is computed by the conjugate gradient method in line with the convergence criterion $C P I[k]-C P I[k-1]<\delta$, where $k$ is the iteration number and $\delta$ is the scalar constant.

Redundant force control. Another approach is a redundant force control with a multi-points interaction [14] given by

$$
\boldsymbol{\tau}=\boldsymbol{K}_{1} \boldsymbol{J}^{T}\left(\boldsymbol{F}_{d}-\boldsymbol{F}\right)+\boldsymbol{K}_{2} \int \boldsymbol{J}^{T}\left(\boldsymbol{F}_{d}-\boldsymbol{F}\right) d t+\boldsymbol{g}
$$

where $\boldsymbol{\tau}=\left(\boldsymbol{\tau}_{\text {arm }}{ }^{T}, \boldsymbol{\tau}_{\text {hand }}{ }^{T}\right)^{T} \in R^{21}$ is a joint torque vector whose sub vectors are an arm joint torque vector $\boldsymbol{\tau}_{\text {arm }} \in R^{6}$ and a hand joint torque vector $\boldsymbol{\tau}_{\text {hand }}=\left(\boldsymbol{\tau}_{\text {thumb }}{ }^{T}{ }^{2}{ }_{\text {index }}{ }^{T}, \cdots, \boldsymbol{\tau}_{\text {little }}{ }^{T}\right)^{T} \in R^{15}, \quad \boldsymbol{J} \in R^{21 \times 15}$ is a kinematic Jacobian of HIRO II, $\boldsymbol{F}=\left(\boldsymbol{F}_{\text {thumb }}{ }^{T}, \boldsymbol{F}_{\text {index }}{ }^{T}, \cdots, \boldsymbol{F}_{\text {little }}{ }^{T}\right)^{T} \in R^{15}$ is a force vector whose sub vector is a force vector at the fingertip, $\boldsymbol{F}_{d}$ is a desired force vector, $\boldsymbol{K}_{l}$ is a force feedback gain matrix, $\boldsymbol{K}_{2}$ 
is a force integral feedback gain matrix and $\boldsymbol{g}$ is a gravity compensator. Most of redundant force control [17]-[18] has been examined only on a single constrained point at the manipulator's endeffector. This redundant force control works at multi-contacting points. Each finger joint is controlled so as to follow the desired finger force independently. The arm joint is controlled so as to follow the desired force and moment at the hand bases, which are generated by the desired finger forces.

\section{Physical simulation including frictional force and moment}

When an object is manipulated by the operator's hand in virtual space, it is desirable that the object's spatial movement be based on physical laws. The constraint and frictional forces which are generated at the contact points between an operator's fingertips and an object surface (see Fig. 5) are important factors in the real world of humans. In general, constraint force is calculated in proportion to the penetration depth of a fingertip touching an object, and frictional force on a tangential plane at the contact point is calculated in proportion to the constraint force. As a physical phenomenon, there are two friction states at a contact point: a static friction state without sliding and a dynamic friction state with sliding. Some researches on friction models of haptic rendering have been carried out [19]-[21]. For example, W. S. Harwin et al. [20] have presented a method based on god-objects [21] and friction cones which permits the computation of both static and dynamic forces. In this method, the friction is calculated in proportion to the penetration depth of fingertips in regard to a virtual object and also as a haptic interface point moves to the edge of a friction cone in cases where the haptic interface point lies outside the friction cone (see Fig. 6).

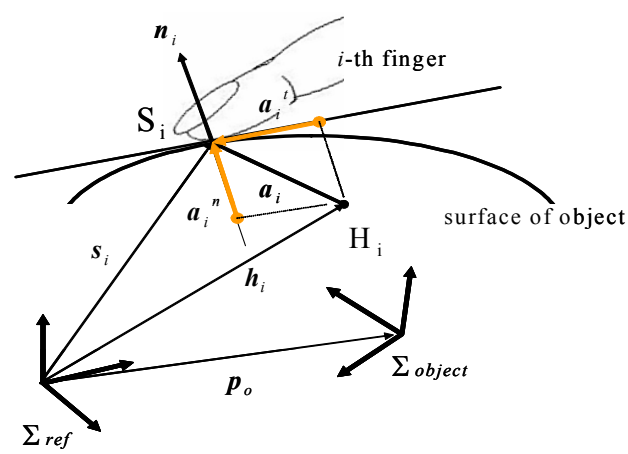

Fig. 5 Constraint force and frictional force

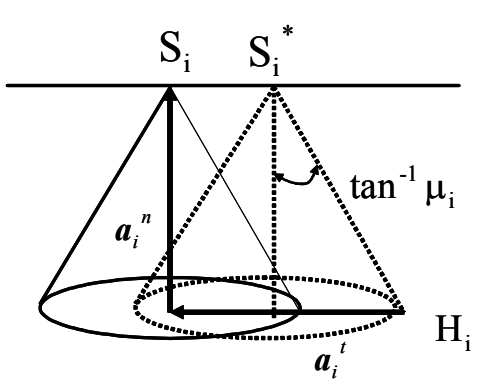

Fig.6 Movement of contact point

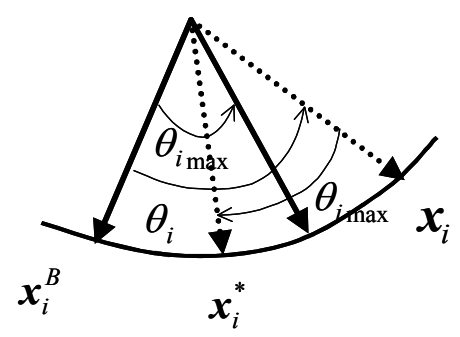

Fig.7 Movement of $\boldsymbol{x}_{i}{ }^{B}$

If the fingertip is spherical and the spot shape is circular, the analytic frictional moment is proportional to the normal contact force [22]. A computational method for a frictional moment proportional to a relative torsion angle and constraint force has been presented [23]. The relative torsion angle of the $i$-th finger is represented as a rotational angle at a contact point $\mathrm{C}_{\mathrm{i}}$ around a normal axis $\boldsymbol{n}_{i}$. We can set up a fingertip coordinate system $\Sigma_{i}{ }^{B}=\left\{\boldsymbol{x}_{i}{ }^{B}, \boldsymbol{y}_{i}{ }^{B}, \boldsymbol{z}_{i}{ }^{B}\right\}$ at the contact point $\mathrm{C}_{\mathrm{i}}$ for the $i$-th fingertip, where $\boldsymbol{z}_{i}^{B}$ coincides with the normal direction of the object surface at the contact point, and the $\boldsymbol{x}_{i}^{B} \boldsymbol{y}_{i}^{B}$-plane coincides with the tangential plane at the contact point. At the same time, an object coordinate system $\Sigma_{i}=\left\{\boldsymbol{x}_{i}, \boldsymbol{y}_{i}, \boldsymbol{z}_{i}\right\}$ at the contact point $\mathrm{C}_{\mathrm{i}}$ of the object is superposed on $\Sigma_{i}{ }^{B}$. When the two coordinate systems move spatially, a rotational angle of the $\boldsymbol{x}_{i}$ axis about the $\boldsymbol{x}_{i}^{B} \times \boldsymbol{x}_{i}$ axis is $\theta_{i}^{*}=\sin ^{-1}\left(\left\|\boldsymbol{x}_{i}^{B} \times \boldsymbol{x}_{i}\right\|\right)$. The relative torsion angle between the fingertip and object at the $i$-th contact point is given by

$$
\theta_{i}=\theta_{i}^{*}\left(\boldsymbol{n}_{i}^{T} \frac{\boldsymbol{x}_{i}^{B} \times \boldsymbol{x}_{i}}{\left\|\boldsymbol{x}_{i}^{B} \times \boldsymbol{x}_{i}\right\|}\right)
$$

The static frictional moment is computed by 


$$
\boldsymbol{m}_{i}=\zeta_{i}\left\|\boldsymbol{f}_{i}^{n}\right\| \boldsymbol{n}_{i}
$$

where $\varsigma_{i}$ is the coefficient of the frictional moment, which is given by $\varsigma_{i}=k_{s} \theta_{i}$ (where $k_{s}$ is a parameter depending on physical characteristics of the fingertip). This satisfies $0 \leq \varsigma_{i} \leq k_{s} \theta_{i \max }$, where $_{\theta_{i \max }}$ is the maximum relative torsion angle at the static frictional moment.

Now let us consider a frictional moment arc with a vertex angle $\theta_{i_{\max }}$, as shown in Fig. 7. When the $\boldsymbol{x}_{i}$ axis lies in the frictional moment arc, that is, $\left|\theta_{i}\right| \leq \theta_{i_{\max }}$, it is a static frictional moment state. When the $\boldsymbol{x}_{i}$ axis lies outside the frictional moment arc, that is, $\left|\theta_{i}\right|>\theta_{i \max }$, it is a dynamic frictional moment state. At the dynamic frictional moment state, the $\boldsymbol{x}_{i}{ }^{*}$-axis obtained by rotating $\boldsymbol{x}_{i}$ around $\boldsymbol{n}_{i}$ with the rotational angle $-\theta_{i \max }$ can be linearly approximated by $\boldsymbol{x}_{i}{ }^{*}=\boldsymbol{x}_{i}-\left(\boldsymbol{x}_{i}-\boldsymbol{x}_{i}^{B}\right) \theta_{i \max } / \theta_{i}$. A new start point of the frictional moment is set by replacing $\boldsymbol{x}_{i}^{*}$ by $\boldsymbol{x}_{i}^{B}$ such that $\boldsymbol{x}_{i}$ lies on the edge of the frictional moment arc. These relationships show that the frictional moment is proportional to the constraint force and the relative torsion angle between the finger and object at the contact point. This computational method has no effect on the contact point and the haptic interface point, which means that computations of the frictional force and friction moment can be done separately. The dynamics of the object can be simulated by taking the frictional force and frictional moment into account.

\section{Haptic rendering with deformable object}

Most high precision techniques for deformable objects tend to be computationally expensive. As a consequence, it is normally very hard to reach high control rates $(>1[\mathrm{KHz}])$. Various solutions have been proposed to solve this problem. Most of the methods deal with intermediate representation or use a local model in the haptic loop to obtain forces at a high update rate [24]-[25]. Nevertheless, all the literature, to our knowledge, is concerned only with a single point contact. When there are multiple points of contact with deformable objects, it is necessary to consider the effect of each point on the other points and how the object should deform based on its interaction with all of the fingers. The use of a local model is insufficient to solve this problem since it is necessary to deal with the whole model or a sufficiently large independent part of it to be able to consider the effects of each finger on the model and on the other fingers. To overcome this difficulty, an "elementary displacement" approach [26] used with a finite element method has been adopted [27].

\section{System architecture}

Separating the haptic processes from the more time-consuming processes has long been a standard technique to protect more sensitive servo loops from the computational burden of collision detection and graphic display. However, most of the implementation tends to involve both graphics and haptic rendering on the same machine and, to make matters worse, to use an approach other than a real-time operating system. Our approach differs as it decouples haptic and graphics rendering, running each on a separate machine. This split is achieved by connecting the haptic device directly to the force server, which tracks the probe of the haptic device and executes the haptic servo loop. The application connects to this force server through a communication channel, retrieving position information from the server and sending the desired forces to it. We use a TCP/IP gigabit communications channel since it offers low latency and high bandwidth. The architecture can be described as distributed and multi-threaded on separate machines; in addition, the requirements for haptics and graphics are well considered. The software side (VR simulation) and the hardware side (the haptic controller) are connected mainly through a high speed network, as shown in Fig. 8. 


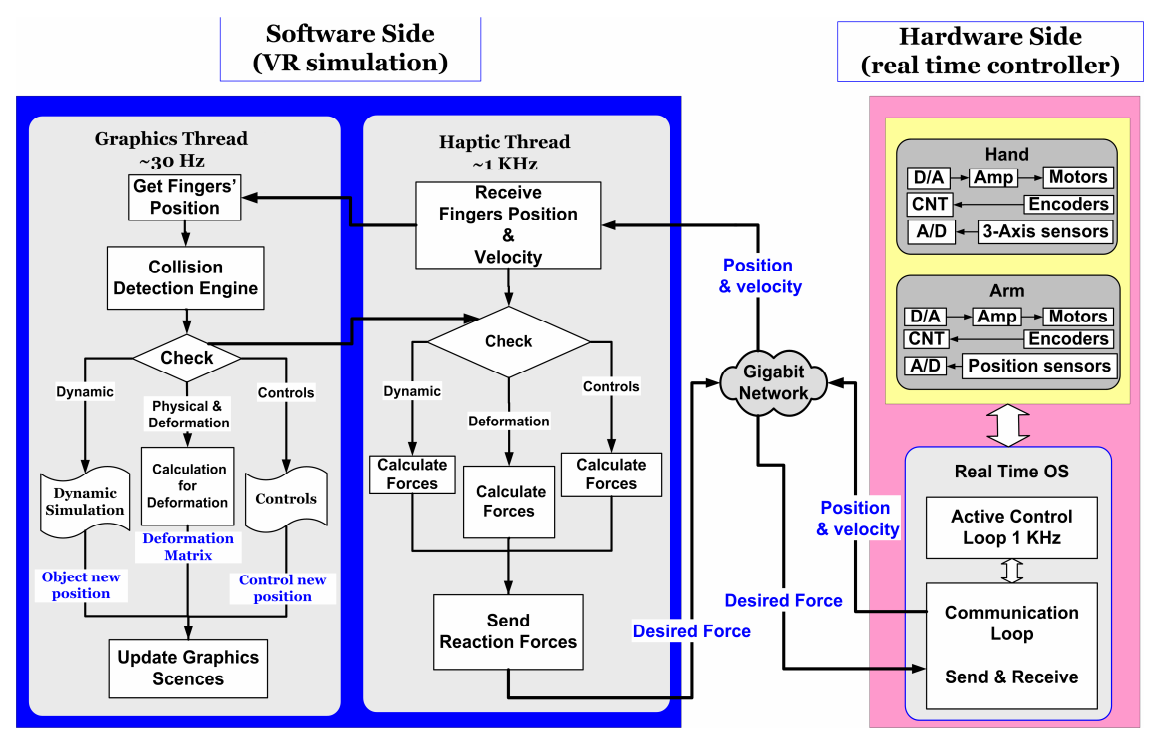

Fig. 8. Software architecture

\section{Application systems}

Future science encyclopedia. Maintaining a stable haptic interaction in VR environments, especially with physically-based deformable objects, has long been an active area of research. Our group has proposed a comprehensive haptic system architecture for haptic rendering, in which a physically-based model using the FEM with a elementary displacement approach was implemented [28]. The Future Science Encyclopedia (FSE) had been developed to verify and demonstrate our proposal at the 2005 World Exposition, Aichi, Japan. Moreover, the work involved testing the proposed architecture and verifying the force and deformation calculation methods. The FSE enables users to experience many different virtual worlds like the scientific, historical, and astronomical. These worlds extend from macrospace to microspace, and involve fantastic worlds from ancient times to the future. The interesting feature of the FSE is that it can be considered a complete VR interface where the user uses only HIRO II to interact and move between and around the scenes, as Fig 9 shows.

VR breast palpation system. A multi-fingers haptic device that enables multiple points of contact is essential in medical education. The sense of touch can be extremely valuable to trained physicians when diagnosing illnesses. With a combination of technologies such as VR and force feedback robots, it is possible to greatly extend the capabilities and effectiveness of training simulators. A simulation can record kinematics, touch, and force feedback for later display to a trainee. From this, the trainee can learn the methodology of a procedure as well as experience the forces that will be encountered when actually performing the procedure. This technology could be used to train medical students before they palpate a real patient and could also be used by trained physicians to improve their skill. There is a strong need for palpation training in detecting subsurface tumors[29]. An important step in a breast modeling sequence involves the incorporation into a model of large deformation effects and the consideration of nonlinear material properties. We adopted a linear model adaptation technique for a nonlinear force update [30] in which the deformations of the model nodes are calculated according to a linear model and the forces of the touched nodes are updated according to experimentally-measured, force-displacement curves.

In general, tissue is heterogeneous and exhibits non-linear, anisotropic elastic and viscous behavior. Therefore, the combination of these qualities quickly becomes complex and requires huge computation efforts. In order to overcome this problem, we simplified the model by neglecting viscous and inertial forces since the movements in a palpation session are slow. Stress-strain relationships in a biological tissue can be determined by experiments where the forces and displacements are recorded. Then, the measured sample points can be used to determine the stress- 
strain relationship of the material by an interpolation function. During a palpation session in real time, a nonlinear contact force at a touched node is calculated from a linear model data as below:

$$
f=f_{\text {lin }}^{\tau}+f_{\text {nonlin }}^{n}
$$

where $f_{\text {lin }}^{\tau}$ is the tangential component of the force that is computed from a linear model, and $f_{\text {nonlin }}^{n}=P\left(f_{\text {lin }}^{\tau}\right)$ is a normal component of the nonlinear force approximated by a polynomial which uses a linear force as an argument. A finite element model of a silicon breast consists of 13,025 tetrahedron and 2,740 nodes. The breast surface is formed by 1,106 triangular faces and 562 nodes directly accessible for palpation. The minimum and maximum lengths of the finite element edge are 4 and $12[\mathrm{~mm}]$ respectively. This provides an operator with sufficient resolution in tumor palpation. Four tumors were modeled as hard, grounded, sphere-shaped inclusions each $3[\mathrm{~cm}]$ in diameter and located at the four breast quarters.

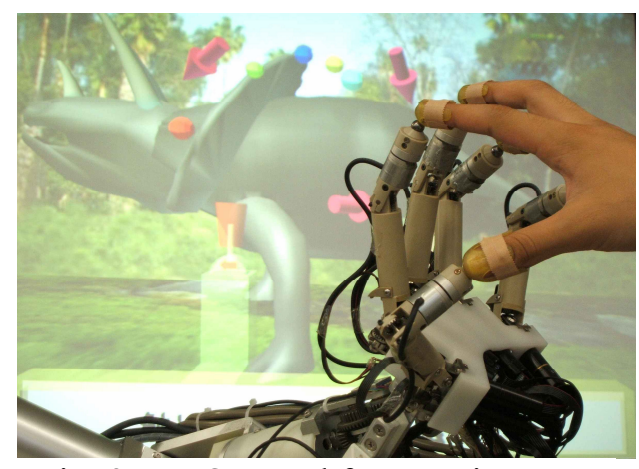

Fig. 9 HIRO II and future science encyclopedia (FSE)

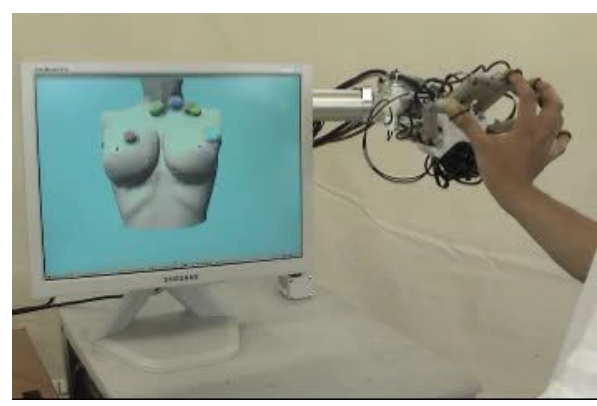

Fig.10 Haptic breast palpation using

\section{Summary}

The design and control method of a five-fingered haptic interface robot named HIRO II has been presented. The interface is placed opposite the human hand to free the operator from its weight and to protect the operator from any possible malfunction. We have introduced the design method of the mechanism, including the optimum finger layout, an interface control taking the redundancy of the mechanism into consideration, physical simulation including frictional force and moment, a haptic rendering with a deformable object, the system architecture consisting of a real-time controller and VR simulation, and two application systems--a future science encyclopedia and VR breast palpation system. This interface can be utilized in a wide range of applications as a master or a slave in both real and virtual environments. Future research will be directed toward implementing the interface in various applications such as in medical training and the teleoperation of an anthropomorphic robot hand.

\section{References}

[1] R.V. Dubey, S.E. Everett, N. Pernalete, and K.A. Manocha: Teleoperation assistance through variable velocity mapping, IEEE Trans. on Robotics and Automation, vol. 17, no. 5, pp. 761-766 (2001).

[2] I. Elhajj, N. Xi, W. K. Fung, Y. H. Liu, W. J. Li, T. Kaga, and T. Fukuda: Haptic information in internetbased teleoperation, IEEE/ASME Trans. on mechatronics, vol. 6, no. 3, pp. 295-304 (2001).

[3] S. Marliere, D. Urma, J. Florens, F. Marchi: Mult-sensorial interaction with a nano-scale phenomenon: the force curve, Proc. of EuroHaptics, pp.246-252 (2004).

[4] C. Basdogan, C. H. Ho, and M. A. Srinivasan: Virtual environments for medical training: graphical and haptic simulation of laparoscopic common bile duct exploration, IEEE/ASME Trans. on Mechatronics, vol. 6, no. 3, pp. 269-286 (2001).

[5] N. A. Langrana, G. Burdea, K. Lange, D. Gomez, and S. Deshpande: Dynamic force feedback in a virtual knee palpation, Artificial Intelligence in Medicine vol. 6, pp. 321-333 (1994). 
[6] H. Kawasaki and T. Hayashi, Force feedback glove for manipulation of Virtual Objects, Jour. of Robotics and Mechatronics, vol. 5, no.1, pp. 79-84 (1993).

[7] Y. Ueda, T. Maeno: Development of a mouse-shaped haptic device with multiple finger inputs, Proc. of IROS2004, pp. 2886-2891(2004).

[8] S. Walairacht, M. Ishii, Y. Koike, and M. Sato: Two-handed multi-fingers string-based haptic interface device, IEICE Trans. on Information and Systems, vol. E84D, No. 3, pp. 365-373 (2001).

[9] M. Bouzit, G. Burdea, G. Popescu, and R. Boian: The rutgers master II - new design force-feed back glove, IEEE/ASME Trans. on Mechatronics, vol. 7, no. 2, pp.256-263 (2002).

[10] Y. Adachi, et. al.: Development of a haptic device for multi fingers by Macro-Micro Structure, Jour. of the Robotics Society of Japan, vol. 20, no. 7, pp. 725-733 (2002).

[11] T. Yoshikawa and A. Nagara: Development and control of touch and force display devices for haptic interface, Proc. of SYROCO'00, pp. 427-432 (2000).

[12] Immersion Corporation: CyberForce, http://www.immersion.com/3d/products/cyber_force.php

[13] H. Kawasaki, J. Takai, Y. Tanaka, C. Mrad, and T. Mouri: Control of multi-fingered haptic interface opposite to human hand, Proc. of IROS2003, pp. 2707-2712 (2003).

[14] H. Kawasaki, T. Mouri, M. O. Alhalabi, Y. Sugihashi, Y. Ohtuka, S. Ikenohata, K. Kigaku, V. Daniulaitis, K. Hamada and T. Suzuki: Development of five-fingered haptic interface: HIRO II, Proc. of ICAT 2005, pp. 209-214 (2005).

[15] T. Mouri, H. Kawasaki, K. Yoshikawa, J. Takai, and S. Ito: Anthropomorphic Robot Hand: Gifu Hand III, Proc. of Int. Conf. ICCAS2002, pp.1288-1293 (2002).

[16] H. Kawasaki and T. Mouri: Design and Control of Five-Fingered Haptic Interface Opposite to Human Hand, IEEE Trans. of Robotics and Automation (Submitted).

[17] Z. X. Peng, and N. Adachi: Compliant motion control of kinematically redundant manipulators, IEEE Trans. on Robotics and Automation, vol. 9, no. 6, pp.831-837 (1993).

[18] D.G. Li, A. A. Goldenberg, and J.W. Zu: A new method of peak torque reduction with redundant manipulators, IEEE Trans. on Robotics and Automation, vol. 13, no. 5, pp.845-853 (1997)

[19] V. Hayward: A New Computational Model of Friction Applied to Haptic Rendering, Proc. of Experimental Robotics VI (2000).

[20] W. S. Harwin and N. Melder: Improved Haptic Rendering for Multi-Finger Manipulation Using Frivtion Cone based God-Objects, Proc. of Eurohaptics Conference (2002).

[21] C. B. Zilles and J. K. Salisbury:A Constraint-based God-object Method for Haptic Display , Proceedings of International Conference on Intelligent Robots and Systems , vol. 3, pp.146151 (1995).

[22] H. Takao et al.: Analysis and Simulation of Grasping/ Manipulation Dynamics by Multifinger Surface, 9th World Congress on Theory of Mechanics and Mechanisms, pp.2272-2276 (1995).

[23] H. Kawasaki, Y. Ohtuka, M.O. Alhalabi, T. Mouri: Haptic Rendering and Perception of Frictional Moment, Proc. of EuroHaptics2006, pp.201-206 (2006).

[24] F. Barbagli, D. Prattichizzo, K. Salisbury: Multirate analysis of haptic interaction stability with deformable objects, Proc. of the 41st IEEE conf. on Decision and Control; pp. 917-922 (2002).

[25] C. Mendoza, C. Laugier: Realistic haptic rendering for highly deformable virtual objects, Proc. of Virtual Reality Conference, pp. 264-269 (2001).

[26] S. Cotin, H. Delingette, and N. Ayache: Real-Time Elastic Deformations of Soft Tissues for Surgery Simulation, IEEE Trans. on Visualization and Computer Graphics, Vol. 5, No. 1, pp. $62-73$ (1999).

[27] V. Daniulaitis, M. O. Alhalabi, H. Kawasaki, Y. Tanaka, T. Hori: Medical palpation of deformable tissue using physics-based model for Haptic Interface RObot (HIRO), Proc. of IROS2004, pp.39073911(2004).

[28] O. Halabi, V. Danulaitis, H. Kawasaki, T. Mouri and Y. Ohtuka: Future Haptic Scirnce Encyclopedia: Realistic Stable Haptic Interaction with Highly Deformable Objects Using HIRO-II, Journal of Robotics and Mechatronics, Vol. 18, No.4, pp. 409-417 (2006).

[29] A. Miller, T. To , C. Baines, et al.: Canadian National Breast Screening Study-2: 13-year results of a randomized trial in women aged 50-59 years. Journal of the National Cancer Institute, Vol. 92, No.18, pp.1490-9 (2000).

[30] M. O. Alhalabi, $\quad$ V. Daniulatis, H. Kawasaki, T. Mouri: Haptic Breast Palpation Using FiveFingers Haptic Interface HIRO-II, The Japanese Journal for medical Virtual reality, Vol. 4 , No.1 , pp.5-11 (2006). 